OPEN

SUBJECT AREAS:

TOPOLOGICAL

INSULATORS

PHASE TRANSITIONS AND CRITICAL PHENOMENA

Received

9 September 2013

Accepted

11 September 2013

Published

27 September 2013

Correspondence and requests for materials should be addressed to

M.E. (ezawa@ap.t.utokyo.ac.jp)

\section{Topological Phase Transition without Gap Closing}

\author{
Motohiko Ezawa', Yukio Tanaka² \& Naoto Nagaosa ${ }^{3,1}$
}

'Department of Applied Physics, University of Tokyo, Hongo 7-3-1, 1 13-8656, Japan, ${ }^{2}$ Department of Applied Physics, Nagoya University, Nagoya 464-8603, Japan, ${ }^{3}$ RIKEN Center for Emergent Matter Science (CEMS), Wako 351-0198, Japan.

Topological phase transition is accompanied with a change of topological numbers. According to the bulk-edge correspondence, the gap closing and the breakdown of the adiabaticity are necessary at the phase transition point to make the topological number ill-defined. However, the gap closing is not always needed. In this paper, we show that two topological distinct phases can be continuously connected without gap closing, provided the symmetry of the system changes during the process. Here we propose the generic principles how this is possible by demonstrating various examples such as $1 \mathrm{D}$ polyacetylene with the charge-density-wave order, 2D silicene with the antiferromagnetic order, 2D silicene or quantum well made of $\mathrm{HgTe}$ with superconducting proximity effects and $3 \mathrm{D}$ superconductor $\mathrm{Cu}$ doped $\mathrm{Bi}_{2} \mathrm{Se}_{3}$. It is argued that such an unusual phenomenon can occur when we detour around the gap closing point provided the connection of the topological numbers is lost along the detour path.

T opological insulator and superconductor are among the most fascinating concepts in physics found in this decade ${ }^{1-7}$. It is characterized by the topological numbers such as the Chern number and the $\mathbb{Z}_{2}$ index. When there are two topological distinct phases, a topological phase transition may occur between them. According to the bulk-edge correspondence, the gap must close at the topological phase transition point since the topological number cannot change its quantized value without gap closing. Note that the topological number is only defined in the gapped system and remains unchanged for any adiabatic process. Alternatively we may think of the edge or surface of the sample in a topological phase. Gapless edge or surface modes appear because the boundary of the sample separates a topological state and the vacuum whose topological numbers are zero. The phenomenon is known as the bulk-edge correspondence. We wonder if a topological phase transition cannot occur without gap closing at all.

The topological classes are classified ${ }^{8}$ by the eigenvalues of $\Theta^{2}, \Xi^{2}$ and $\Pi^{2}$, where $\Theta, \Xi$ and $\Pi$, represent the time-reversal, particle-hole and chiral symmetry operators. There are ten classes, which are separated into two complex and eight real representations. The topological periodic table has been established as in Table I, which classifies all the possible homotopy groups and topological numbers depending on the symmetry and dimensionality of the system. One important fact about this topological periodic table is that the adiabatic connection is possible between the two classes with the difference in dimensions by one ${ }^{9}$. As for the eight real representations, this connection is summarized by the symmetry clock shown in Fig. 1. Namely, considering the Hamiltonian $H(k, r)$ which depends on both the $D$-dimensional real space coordinates $r$ and $d$-dimensional momentum space coordinates $k$, the mapping connecting the neighboring classes in the symmetry clock is possible by adding $r$ - or $k$-dependent Hamiltonian'. This means that the essential dimensionality is $d^{\prime}=d-D$ and the adiabatic connection exists next to each other along the diagonal direction in the topological periodic table. In addition to this diagonal shift, one can consider the horizontal shift, i.e., dimensionality $d^{\prime}$ by introducing the defects such as vortex $(D=1)$, and point defect $(D=2)^{10}$. There are several works on the vertical shift in the periodic table ${ }^{11-15}$. However in these cases, the gap closing is necessary for topological phase transitions.

In this paper, we study the adiabatic connection within the common dimensionality $d^{\prime}$, and the possible change in the topological numbers without closing the gap. We propose two principles. Let the energy spectrum be given by $E_{\rho}(k)$ with a topological phase transition taking place at a critical point $\rho=\rho_{\text {cr }}$ of a certain parameter $\rho$, where the gap closes. Let us assume that we can extend the Hamiltonian to include a new parameter $\Delta_{s}$ so that the energy spectrum is modified as

$$
E(k)= \pm \sqrt{E_{\rho}^{2}(k)+\Delta_{s}^{2}}
$$


Table I | Periodic table for the homotopy group of each class. The rows correspond to the different Altland Zirnbaver (AZ) symmetry classes while the columns distinguish different dimensionalities, which depend only on $d^{\prime}=d-D$ with $d$-dimensional $k$ space and $D$-dimensional real space coordinates

\begin{tabular}{|c|c|c|c|c|c|c|c|c|c|c|c|c|}
\hline \multicolumn{5}{|c|}{ Symmetry } & \multicolumn{8}{|c|}{$d^{\prime}=d-D$} \\
\hline$s$ & $A Z$ & $\Theta^{2}$ & $\Xi^{2}$ & $\Pi^{2}$ & 0 & 1 & 2 & 3 & 4 & 5 & 6 & 7 \\
\hline 0 & $A$ & 0 & 0 & 0 & $\mathbb{Z}$ & 0 & $\mathbb{Z}$ & 0 & $\mathbb{Z}$ & 0 & $\mathbb{Z}$ & 0 \\
\hline 0 & $\mathrm{Al}$ & 1 & 0 & 0 & $\mathbb{Z}$ & 0 & 0 & 0 & $2 \mathbb{Z}$ & 0 & $\mathbb{Z}_{2}$ & $\mathbb{Z}_{2}$ \\
\hline 1 & $\mathrm{BDI}$ & 1 & 1 & 1 & $\mathbb{Z}_{2}$ & $\mathbb{Z}$ & 0 & 0 & 0 & $2 \mathbb{Z}$ & 0 & $\mathbb{Z}_{2}$ \\
\hline 2 & D & 0 & 1 & 0 & $\mathbb{Z}_{2}$ & $\mathbb{Z}_{2}$ & $\mathbb{Z}$ & 0 & 0 & 0 & $2 \mathbb{Z}$ & 0 \\
\hline 3 & DIII & -1 & 1 & 1 & 0 & $\mathbb{Z}_{2}$ & $\mathbb{Z}_{2}$ & $\mathbb{Z}$ & 0 & 0 & 0 & $2 \mathbb{Z}$ \\
\hline 6 & $C$ & 0 & -1 & 0 & 0 & 0 & $2 \mathbb{Z}$ & 0 & $\mathbb{Z}_{2}$ & $\mathbb{Z}_{2}$ & $\mathbb{Z}$ & 0 \\
\hline 7 & $\mathrm{Cl}$ & 1 & -1 & 1 & 0 & 0 & 0 & $2 \mathbb{Z}$ & 0 & $\mathbb{Z}_{2}$ & $\mathbb{Z}_{2}$ & $\mathbb{Z}$ \\
\hline
\end{tabular}

The phase transition point is $\left(\rho_{\mathrm{cr}}, 0\right)$ in the $\left(\rho, \Delta_{s}\right)$ phase diagram. We may detour the point $\left(\rho_{\mathrm{cr}}, 0\right)$ in the phase diagram, along which the gap never closes though a topological phase transition occurs. The second principle is that the topological number should become illdefined by a symmetry change along the above detour. This invalidates requirements of the gap closing when the topological number changes.

\section{Results}

We have explored the generic principles of topological phase transition without gap closing by demonstrating various examples. The first example is a simple one-dimensional model of polyacetylene with reduced symmetry at intermediate states $(\mathrm{BDI} \rightarrow \mathrm{A} \rightarrow \mathrm{BDI})$ by way of the charge-density-wave (CDW). We also present a twodimensional example of silicene with the antiferromagnet (AF) order as another model with symmetry reducing. We then present three models with enhanced symmetry at intermediate state (AII $\rightarrow$ DIII $\rightarrow$ AII) by way of introducing the superconducting (SC) order ${ }^{8}$. They are silicene and quantum well made of $\mathrm{HgTe}$ as two-dimensional models, and superconductor $\mathrm{Cu}$ doped $\mathrm{Bi}_{2} \mathrm{Se}_{3}$ as a three-dimensional model.

Polyacetylene with CDW order. We start with presenting a wellknown one-dimensional example of polyacetylene with the CDW order from a new light. Polyacetylene belongs to the class BDI. With including the CDW order, the class change occurs into A by breaking the time-reversal and particle-hole symmetries simultaneously. We now show that there are two ways of topological phase transitions, one (BDI $\rightarrow$ BDI) with gap closing and the other $(\mathrm{BDI} \rightarrow$ $\mathrm{A} \rightarrow \mathrm{BDI})$ without gap closing.

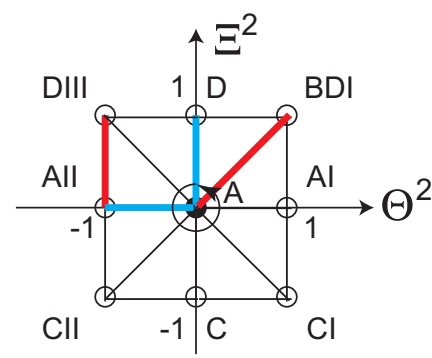

Figure $1 \mid$ Topological classes and possible class changes. The horizontal axis is $\Theta^{2}=0, \pm 1$, which represents the time-reversal symmetry, while the vertical axis is $\Xi^{2}=0, \pm 1$, which represents the particle-hole symmetry. The eigenvalue 0 means the absence of the symmetry. We present examples of class changes by bold lines that occur without gap closing. AIII resides with the same position as A. The red (blue) lines indicate the topological phase transition without (with) gap closing.
Effective Hamiltonian. Polyacetylene is a bipartite system with one unit cell made of $A$ and $B$ sites. The bipartiteness introduces a pseudospin. We neglect the spin degree of freedom. The tight-binding model is given by ${ }^{16-20}$

$$
H_{\text {poly }}=d_{x} \tau_{x}+d_{y} \tau_{y}
$$

in the momentum space, where $\tau_{i}$ is the Pauli matrix acting on the pseudospin $\Psi=\left\{\psi_{A}, \psi_{B}\right\}$, and

$$
d_{x}=t+\delta+(t-\delta) \cos k, \quad d_{y}=(t-\delta) \sin k,
$$

where $k$ is the momentum $(0 \leq k<2 \pi), t$ is the mean transfer integral, $\delta$ is the dimerization of the transfer integral. The energy spectrum is given by

$$
E_{\text {poly }}(k)= \pm 2 \sqrt{t^{2} \cos ^{2} \frac{k}{2}+\delta^{2} \sin ^{2} \frac{k}{2}} .
$$

The band gap locates at $k=\pi$, and is given by $2\left|E_{\text {poly }}(\pi)\right|=2|\delta|$. We consider polyacetylene with a finite length. We show the band structure in Figs. 2(c1), (c2), (c3). For $\delta<0$, the system is in the topological phase, as is evidenced by the presence of the gapless edge states in Fig. 2(c1). As $|\delta|$ increases, the gap decreases, and vanishes at $\delta=0$ as in Fig. 2(c2). For $\delta>0$, the gap opens again but no gapless modes appear as in Fig. 2(c3): Hence, it is in the trivial phase. Thus, the topological phase transition occurs with gap closing at $\delta=0$.

We proceed to introduce CDW to polyacetylene. We assume it to generate the site-energy difference $m$ between the $A$ and $B$ sites. The Hamiltonian is modified as

$$
H_{\mathrm{CDW}}=H_{\text {poly }}+m \tau_{z} \text {. }
$$

The energy spectrum is modified to be

$$
E_{\mathrm{CDW}}(k)= \pm \sqrt{\left[E_{\text {poly }}(k)\right]^{2}+m^{2}},
$$

which is of the form (1). The gap does not close when $m \neq 0$.

Phase diagram. We explore the topological phase diagram in the ( $\delta$, $m$ ) plane in Fig. 2(a). We show the band gap as a function of the electric field $\delta$ and the CDW gap $m$ in Fig. 2(b). It is intriguing that the gapless point exists only at one isolated point $(0,0)$ in the phase diagram. We consider two paths connecting the topological state at $(\delta, 0)$ with $\delta<0$ and a trivial state at $(\delta, 0)$ with $\delta>0$ shown in the phase diagram. In Fig. 2(c) we show the energy spectrum of finite chain at typical points.

We have already studied the first path along the $\delta$ axis. As the second path, we first move along the $m$ axis. As changing $\delta$, there is no gap closing even at $\delta=0$ due to the CDW gap: See Fig. 2(c5). When $\delta$ exceeds 0 as in Fig. 2(c6) we remove the CDW order. The 
(a) phase diagram

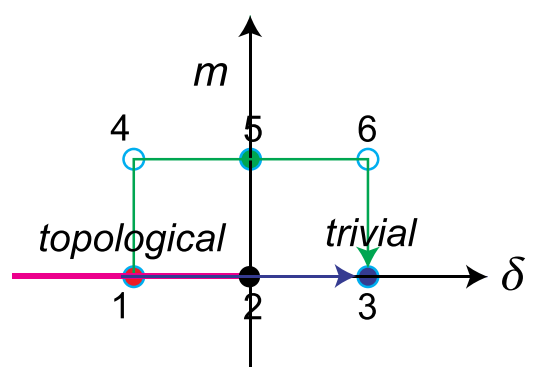

(b) band gap

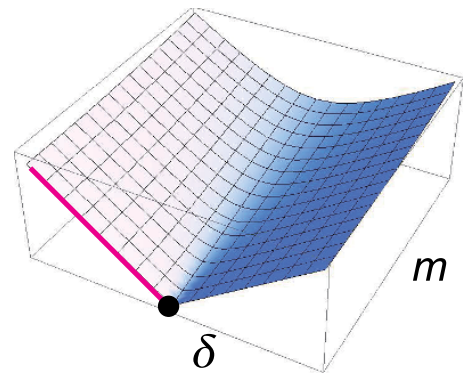

(c) band structure of finite chain

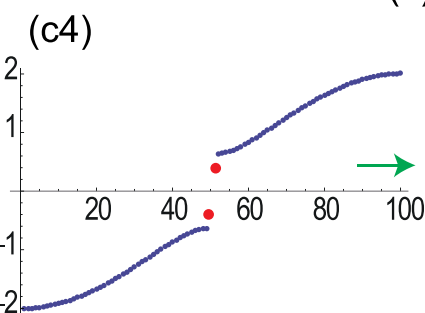

(c1) (c5)

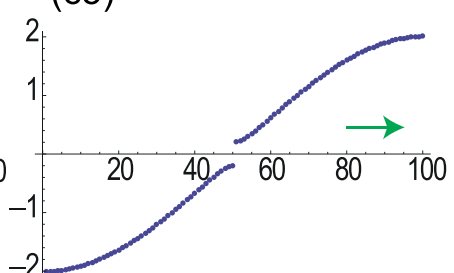

(c2)
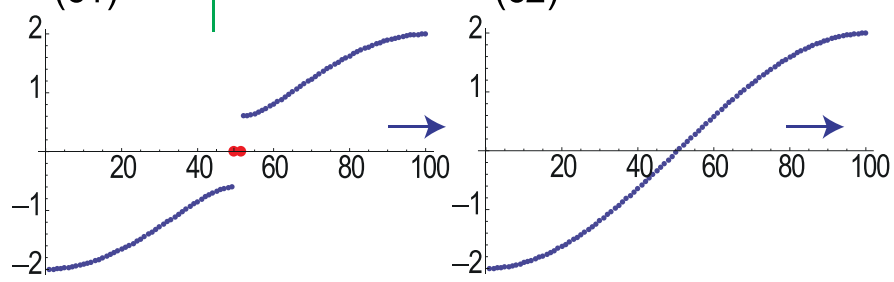

(c6)

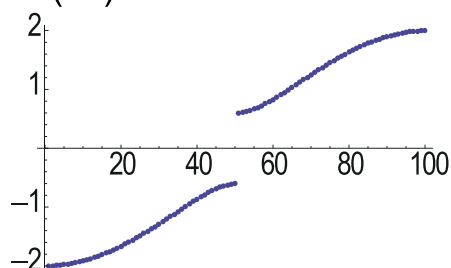

(c3)

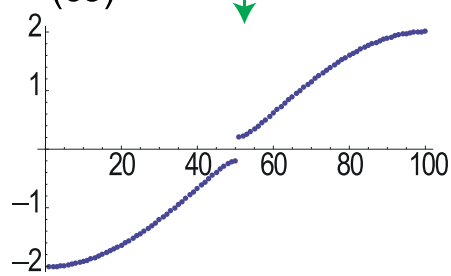

Figure $2 \mid$ Phase diagram and band gap of polyacetylene. (a) Topological phase diagram in the $(\delta, m)$ plane. The horizontal axis is the dimerization $\delta$ and the vertical axis is the CDW gap $m$. The band gap closes at the point denoted by a filled circle. The system is in topological state on the red line along the $\delta$ axis. Circles show points where the energy spectrum is calculated for finite chains in (c). (c1) (c6) Energy spectrum of a finite chain in each point in the phase diagram. The vertical axis is the energy in unit of $t$ and the horizontal axis is the numbering of eigenvalues.

resultant phase is a trivial insulator, as is given by Fig. 2(c3) on the $\delta$ axis. Along this process the gap never closes. This is an explicit example of a topological phase transition without gap closing.

Topological analysis. We analyze the topological number. For this purpose we define

$$
\begin{aligned}
N_{\text {wind }}(m) & =\frac{1}{2 \pi} \int_{0}^{2 \pi} d k\left[n_{x} \partial_{k} n_{y}-n_{y} \partial_{k} n_{x}\right] \\
& =\frac{1}{2}-\frac{t \delta+m^{2}}{2 \sqrt{t^{2}+m^{2}} \sqrt{\delta^{2}+m^{2}}},
\end{aligned}
$$

where $\left(n_{x}, n_{y}, n_{z}\right)=\left(d_{x}, d_{y}, m\right) / \sqrt{d_{x}^{2}+d_{y}^{2}+m^{2}}$ is the normalized vector, which we illustrate in Fig. 3(a).

When $m=0$, the pseudospin is confined in the $x y$ plane, and the homotopy class is $\pi_{1}\left(S^{1}\right)=\mathbb{Z}$. Correspondingly, the quantity (7) takes only two values; $N_{\text {wind }}=1$ for $\delta<0$, and $N_{\text {wind }}=0$ for $\delta>$ 0 . It is the winding number, as explained in Fig. 3(a). Indeed, the system is topological for $\delta<0$, and trivial for $\delta>0$.

On the other hand, when the CDW is present $(m \neq 0)$, the pseudospin acquires the $z$ component, and the homotopy class changes to the trivial class $\pi_{1}\left(S^{2}\right)=0$. The quantity (7) is no longer the quantized to be an integer. Indeed, we can continuously change $N_{\text {wind }}(m)$ from $N_{\text {wind }}(m)=1$ to $N_{\text {wind }}(m)=0$ as we move in the $(\delta, m)$ plane: See Fig. 3(b).

Silicene with AF order. We next present a two-dimensional example of silicene with the AF order as another model with symmetry reducing. Silicene is a honeycomb structure made of silicone atoms. It is a quantum spin-Hall (QSH) insulator ${ }^{21}$, and belongs to the class AII. When we introduce the AF order in the $z$ axis, the class changes into A, but it is still a topological insulator. We call it spinChern insulator because the time-reversal symmetry is broken. We show that there are two ways of topological phase transitions between the QSH insulator and the trivial insulator with and without gap closing. (a)

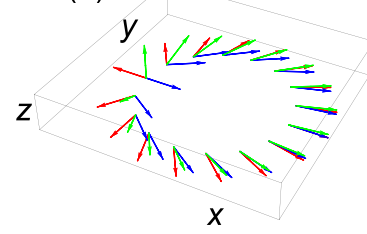

(b)

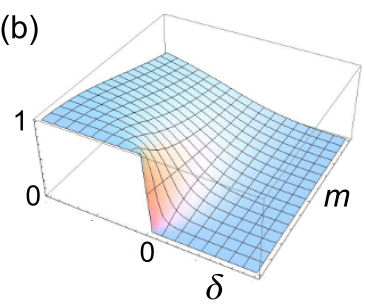

Figure $3 \mid$ Bipartite pseudospin and winding number in polyacetylene. (a) Bipartite pseudospin in a polyacetylene chain in the momentum space $(0 \leq k \leq 2 \pi)$. The pseudospin is confined within the $(x, y)$ plane. The red and blue arrows correspond to the pseudospin configurations $\left(n_{x}, n_{y}\right)$ for $\delta$ $<0$ and $\delta>0$, respectively. They belongs to different homotopy classes with the winding number $N_{\text {wind }}=1$ and 0 . The pseudospin is allowed to have the $z$ component in the presence of the CDW order, as indicated by the green arrows. It connects two different classes continuously. (b) The winding number $N_{\text {wind }}$ in the $(\delta, m)$ space. It changes suddenly at the phase transition point $\delta=0$ along the $\delta$ axis, but smoothly when the point is detoured. 
Low-energy Dirac theory. We analyze the physics of electrons near the Fermi energy, which is described by Dirac electrons near the $K$ and $K^{\prime}$ points. We also call them the $K_{\eta}$ points with the valley index $\eta$ $= \pm$. We introduce the AF order $m_{z}$ along the $z$ direction. The lowenergy Dirac theory reads ${ }^{13,22,23}$

$$
H_{\eta}^{\mathrm{AFz}}=\hbar v_{\mathrm{F}}\left(\eta k_{x} \tau_{x}+k_{y} \tau_{y}\right)+\eta \lambda_{\mathrm{SO}} \sigma_{z} \tau_{z}-m_{z} \sigma_{z} \tau_{z},
$$

where $\sigma_{a}$ and $\tau_{a}$ with $a=x, y, z$ are the Pauli matrices of the spin and the sublattice pseudospin, respectively. The first term arises from the nearest-neighbor hopping, where $v_{\mathrm{F}}=\frac{\sqrt{3}}{2 \hbar} a t=5.5 \times 10^{5} \mathrm{~m} / \mathrm{s}$ is the Fermi velocity with the lattice constant $a=3.86 \AA$. The second term is the intrinsic spin-orbit interaction with $\lambda_{\mathrm{SO}}=3.9 \mathrm{meV}$. The third term represents the AF order. We have neglected the Rashba interaction since its existence does not modify the essential part of the physics. We present the Hamiltonian containing it in Supplementary Information.

The system undergoes a topological phase transition as the AF order $m_{z}$ changes ${ }^{13}$. The energy spectrum is given by

$$
E^{\mathrm{AFz}}(k)= \pm \sqrt{\left(\hbar v_{\mathrm{F}}\right)^{2} k^{2}+\left(\eta \lambda_{\mathrm{SO}}-m_{z}\right)^{2}}
$$

The system is a topological insulator for $\left|m_{z}\right|<2 \lambda_{S O}$ with the gap $2\left|\lambda_{S O}-m_{z}\right|$, as is evidenced by the emergence of gapless edge modes based on the bulk-edge correspondence: See Fig. 4(c1). As $\eta m_{z}$ changes at the $K_{\eta}$ point, the gap decreases and closes at the critical point $m_{z}=\eta \lambda_{\text {so, }}$ as in Fig. 4(c2). Then, the gap opens again, but there appear no longer gapless edge modes as in Fig. 4(c3), indicating that the system is in the trivial phase. The Chern and spin-Chern numbers are calculated to be

\section{(a) phase diagram}

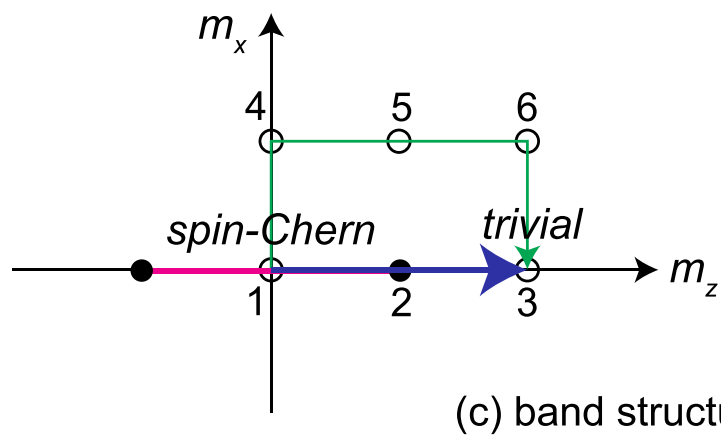

(c4)

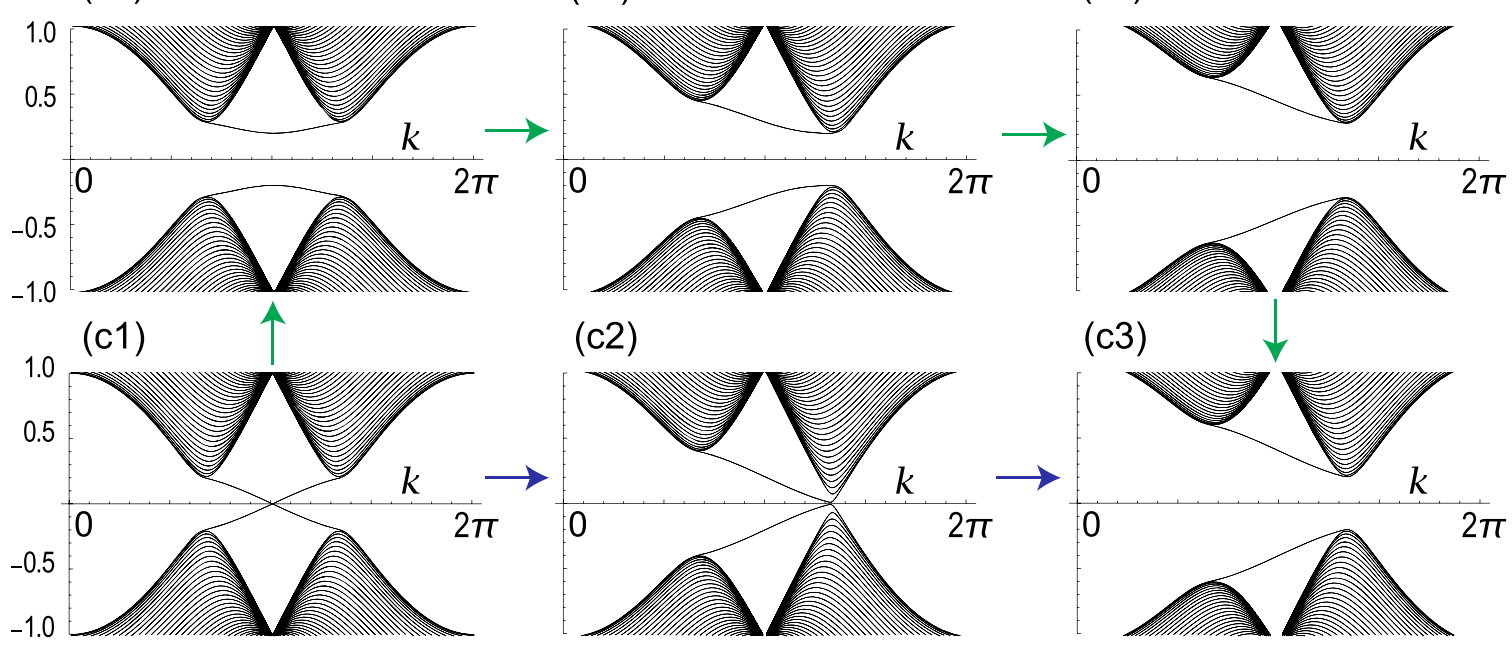

$$
\left(\mathcal{C}, \mathcal{C}_{\text {spin }}\right)=\left\{\begin{array}{l}
(0,1) \text { for }\left|m_{z}\right|<\lambda_{\mathrm{sO}} \\
(0,0) \text { for }\left|m_{z}\right|>\lambda_{\mathrm{sO}}
\end{array}\right.
$$

This is a typical example of a topological phase transition with gap closing.

Let us now introduce the AF order additionally in the $x$ and $y$ directions. The low-energy Dirac theory is given by

$$
H_{\eta}^{\mathrm{AF}}=H_{\eta}^{\mathrm{AFz}}-\left(m_{x} \sigma_{x}+m_{y} \sigma_{y}\right) \tau_{z} .
$$

The energy spectrum is given by

$$
E^{\mathrm{AF}}(k)= \pm \sqrt{\left[E^{\mathrm{AFz}}(k)\right]^{2}+m_{x}^{2}+m_{y}^{2}}
$$

which is of the form (1). The band gap locates at $k=0$, and is given by $2\left|E_{\mathrm{si}}(0)\right|=2 \sqrt{\left(\eta \lambda_{\mathrm{SO}}-m_{z}\right)^{2}+m_{x}^{2}+m_{y}^{2}}$. It closes only at $\left(m_{x}, m_{y}, m_{z}\right)$ $=\left(0,0, \eta \lambda_{\mathrm{SO}}\right)$.

Phase diagram. We explore the phase diagram in the $\left(m_{z}, m_{x}\right)$ plane with $m_{y}=0$ in Fig. 4(a). We show the band gap as a function of the AF orders $m_{z}$ and $m_{x}$ in Fig. 4(b). The gapless points exist only at two isolated points $\left(\eta \lambda_{\mathrm{SO}}, 0\right)$ in the phase diagram. We consider two paths connecting the QSH state at the origin and a trivial state at $\left(m_{z}, 0\right)$ with $m_{z}>\lambda_{\text {SO }}$ shown in the phase diagram. In Fig. 4(c) we show the band structure of silicene with edges at typical points. Along the detour the gap never closes. Hence we have shown that there are two ways of topological phase transitions with and without gap closing.

Topological analysis. Silicene is a topological insulator characterized by the Chern number and the $\mathbb{Z}_{2}$ index. It is to be noted that the $z$ axis

\section{(b) band gap}

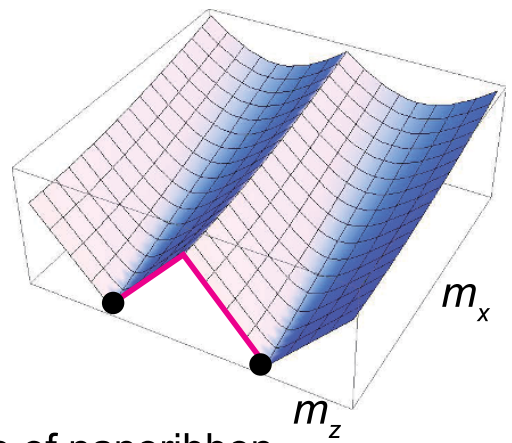

(c5)

\section{(c6)}


has been chosen by the intrinsic SO interaction in the Hamiltonian (8). As far as the AF order is along the $z$ axis, there exists a rotational symmetry around the $z$ axis. The system is the sum of two decoupled systems of $s_{z}=1 / 2$ and $-1 / 2$, and their respective Chern numbers are the topological numbers in this case. Equivalently, one can define the sum (Chern number) and the half of the difference (spin-Chern number) of these two Chern numbers. Note that the $\mathbb{Z}_{2}$ index is well defined when the time-reversal symmetry is present while the spinChern number is well defined when the spin $s_{z}$ is a good quantum number. They are equal mod 2 when both of them are well defined ${ }^{24}$.

When $m_{x}=m_{y}=0$, the spin-Chern number $\mathcal{C}$ is well-defined. The topological phase is indexed by $\mathcal{C}_{\text {spin }}=1$. However, when we introduce $m_{x}$, the AF direction cants, and the spin-Chern number becomes ill-defined. The system becomes a trivial insulator for $m_{x}$ $\neq 0$ or $m_{y} \neq 0$. After we make a detour around the critical point, we decreases $m_{x}$ until $m_{x}=0$, where the spin-Chern number becomes well-defined again. However, the system is in the trivial phase with $\mathcal{C}_{\text {spin }}=0$ when $m_{z}>\lambda_{\text {so. }}$

Helical edge states with AF order. It is quite interesting that the QSH insulator becomes a trivial insulator as soon as $m_{x}$ is introduced. (We assume $m_{z}=0$ for simplicity.) We are able to construct an effective low energy theory to explain how such a transition occurs. The helical edges are described by the $4 \times 4$-matrix Hamiltonian given by

$$
H_{\text {edge }}=\left(\begin{array}{cc}
\left(\lambda_{\mathrm{SO}} / t\right) \hbar v_{\mathrm{F}} k \sigma_{z} & m_{x} \sigma_{x} \\
m_{x} \sigma_{x} & -\left(\lambda_{\mathrm{SO}} / t\right) \hbar v_{\mathrm{F}} k \sigma_{z}
\end{array}\right) .
$$

It is diagonalized as

$$
E_{\mathrm{BdG}}(k)=\sqrt{\left(\frac{\lambda_{\mathrm{SO}}}{t} \hbar v_{\mathrm{F}} k\right)^{2}+m_{x}^{2}} .
$$

The Hamiltonian describes two edge modes crossing at $k=0$ for $m_{x}$ $=0$. As soon as $m_{x} \neq 0$, the level crossing turns into the level anticrossing, with open gap. The gap monotonously increases as $\left|m_{x}\right|$ increases. The system is topological for $m_{x}=0$ and is trivial for $m_{x}$ $\neq 0$.

Silicene with SC order. We then present three models with symmetry increasing transitions (AII $\rightarrow$ DIII) by way of superconducting proximity effect ${ }^{25-30}$. The system is in the class AII without the SC order, and the gap closing occurs at the topological phase transition point separating the two phases with $\mathbb{Z}_{2}=1$ and 0 . However, we are able to make a topological phase transition (AII $\rightarrow$ DIII $\rightarrow$ AII) to occur without gap closing by switching on and off the SC order.

The first model is silicene with the SC order, which might be experimentally achieved by silicene synthesized on the Ir substrate, which has recently been found ${ }^{31}$. Its prominent feature is that $\mathrm{Ir}$ is superconducting at $0.1 \mathrm{~K}$. This opens a natural way to fabricate superconducting proximity effects on to silicene by cooling down the system made of silicene together with the Ir substrate.

Low-energy Dirac theory. We analyze silicene by applying external electric field perpendicular to the sheet. The effective Dirac Hamiltonian in the momentum space reads ${ }^{22,23,32}$

$$
H_{\eta}=\hbar v_{\mathrm{F}}\left(\eta k_{x} \tau_{x}+k_{y} \tau_{y}\right)+\lambda_{\mathrm{SO}} \sigma_{z} \eta \tau_{z}-V \tau_{z} .
$$

The third term is the staggered potential term $V$ induced by the electric field.

The system exhibits a topological phase transition from a QSH insulator to a trivial insulator as $|V|$ increases $^{32}$. The energy spectrum is given by

$$
E_{\mathrm{si}}(k)= \pm \sqrt{\left(\hbar v_{\mathrm{F}}\right)^{2} k^{2}+\left(\eta s_{z} \lambda_{\mathrm{SO}}-V\right)^{2}}
$$

The band gap locates at $k=0$, and is given by $2\left|E_{\mathrm{si}}(0)\right|=2 \mid \eta s_{z} \lambda_{\mathrm{SO}}-$ $V \mid$. It closes at $V= \pm \lambda_{\text {SO }}$. The QSH state has gapless edge states as in

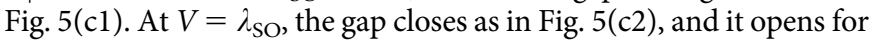
$V>\lambda_{\text {so }}$ as in Fig. 5(c3). The Chern and spin-Chern numbers are calculated to be

$$
\left(\mathcal{C}, \mathcal{C}_{\text {spin }}\right)=\left\{\begin{array}{l}
(0,1) \text { for }|V|<\left|\lambda_{\text {so }}\right| \\
(0,0) \text { for }|V|>\left|\lambda_{\text {so }}\right|
\end{array},\right.
$$

This is a typical example of a topological phase transition with gap closing.

We assume that Cooper pairs are formed by mixing electrons at the $K$ and $K^{\prime}$ points and condense in silicene by attaching the s-wave SC. We present the BCS Hamiltonian and the associated BdG Hamiltonian in Supplementary Information.

It is straightforward to diagonalize the BdG Hamiltonian,

$$
E_{\mathrm{BdG}}(k)= \pm \sqrt{\left[E_{\mathrm{si}}(k)\right]^{2}+\Delta_{s}^{2}}
$$

which is of the form (1). The band gap locates at $k=0$, and it closes at $\left( \pm \lambda_{\mathrm{SO}}, 0\right)$ in the $\left(V, \Delta_{s}\right)$ plane.

Phase diagram. We explore the phase diagram in the $\left(V, \Delta_{s}\right)$ plane in Fig. 5(a). We show the band gap as a function of the electric field $V$ and the SC gap $\Delta_{s}$ in Fig. 5(b). The gapless points exist only at two isolated points $\left( \pm \lambda_{\mathrm{SO}}, 0\right)$ in the phase diagram. We consider two paths connecting the QSH state at the origin and a trivial state at $(V, 0)$ with $V>\lambda_{\text {so }}$ shown in the phase diagram. In Fig. 5(c) we show the band structure of silicene with edges at typical points.

As to the second path, we first move along the $\Delta_{s}$ axis from the origin in the phase diagram. Namely, we cool down the sample below the critical temperature of superconductivity. As soon as the critical temperature is passed, a topological class change occurs by adding the particle-hole symmetry associated with the Cooper-pair condensation. The SC gap mixes the helical edge modes, resulting in the disappearance of gapless edge modes, and the system becomes trivial [Fig. 5(c4)]. The mechanism how the helical edge modes disappear is precisely the same as we demonstrated before: See Eq.(14). After the detour we warm up the sample. The superconductivity disappears. The resultant phase is a trivial insulator, as is given by Fig. 5(c3) on the $V$ axis. Along this process the gap never closes.

Let us study this class change more in detail to confirm that it does not involve the gap closing. For this purpose we analyze a system where SC is attached on one-half of the system. It is important to demonstrate whether there emerge edge states between the boundary of the two regions ${ }^{25,33}$. The region without SC is in the QSH phase, while the superconducting region is in the trivial phase. We may alternatively calculate the band structure of silicene in torus geometry with the SC order introduced to one-half side of a cylinder [Fig. 6(a)]. Note that there are no edge states in silicene in torus geometry even in the QSH phase. The band structure is well known ${ }^{34}$ and given in Fig. 6(b). Once the superconducting gap is introduced partially as in Fig. 6(a), two boundaries appear between the SC and normal regions. We naively expect the emergence of gapless edge modes along each boundary due to the bulk-edge correspondence, as is the case ${ }^{34}$ between the QSH phase and the trivial phase. Nevertheless, we find no gapless edge states to appear as in Fig. 6(c). This reflects the fact that the topological phase changes between $\Delta_{s}=0$ and $\Delta_{s} \neq 0$ undergoes without gap closing.

Topological analysis. We next search for the reason why a topological phase transition can occur without gap closing as soon as the superconductor gap $\Delta_{s}$ is introduced. For this purpose we investigate the topological charges of the system. With the superconducting proximity effects, silicene belongs to the class DIII. Thus the topological class change occurs from AII to DIII as soon as the superconductor gap $\Delta_{s}$ is introduced, i.e., by adding the particle-hole and chiral symmetries. Both classes AII and DIII are characterized by the $\mathbb{Z}_{2}$ 
(a) phase diagram

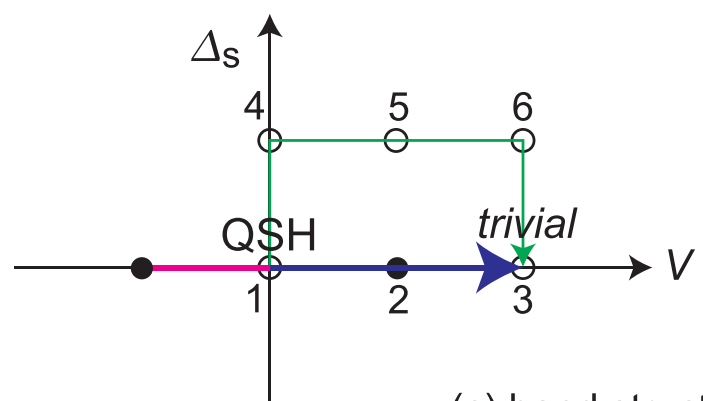

(b) band gap and/or SC gap

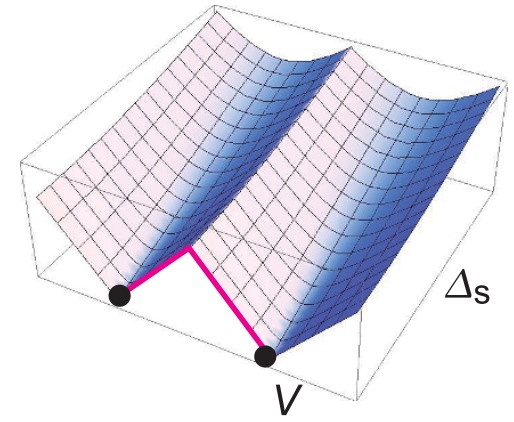

(c) band structure of nanoribbon

(c4)

(c5)

(c6)

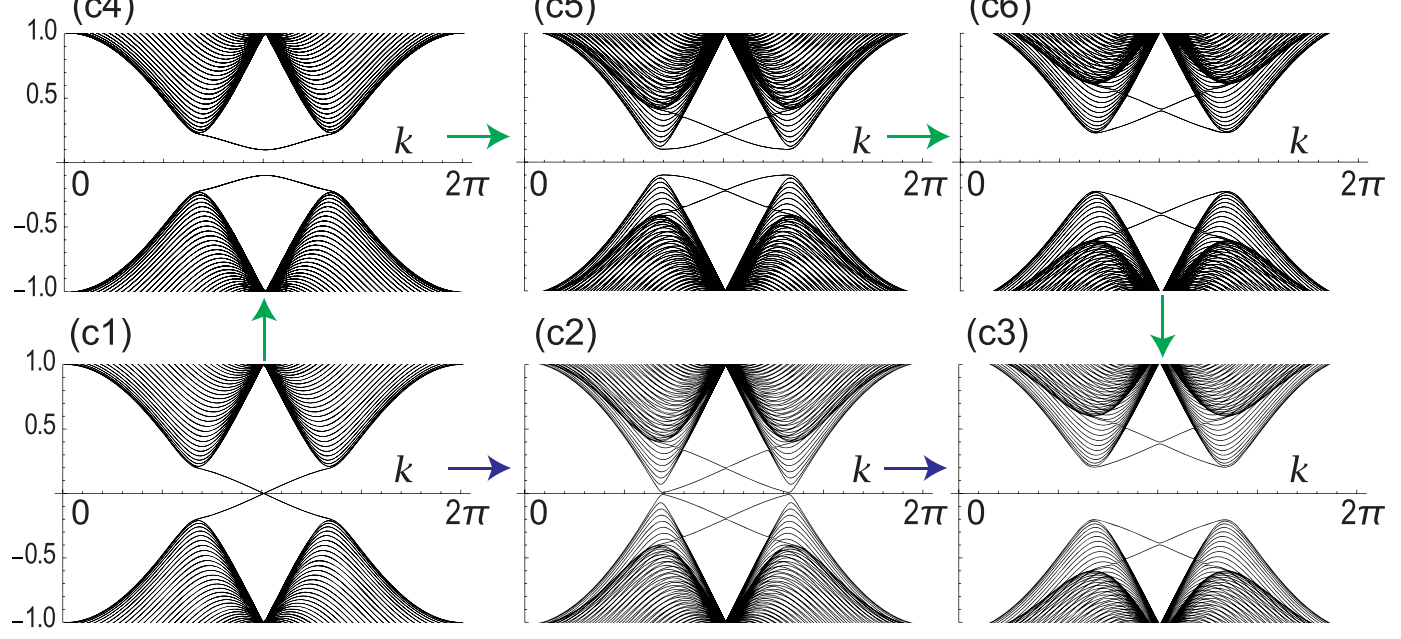

Figure $5 \mid$ Phase diagram and band gap of silicene with SC order. (a) Topological phase diagram in the $\left(V, \Delta_{s}\right)$ plane. The horizontal axis is the electric field $V$ and the vertical axis is the superconducting gap $\Delta_{s}$. (c) We have set $\lambda_{\text {so }}=0.2 t$ for illustration. See also the caption of Fig. 3 .

indices. However they are different objects. Consequently, although we have given the phase diagram in Fig. 5(a), each domain is indexed by different topological indices.

Since the spin $s_{z}$ is a good quantum number along the $E$ axis in the phase diagram, the $\mathbb{Z}_{2}$ index is essentially the spin-Chern number for $\Delta_{s}=0$. The latter counts the numbers of up-spin electrons and downspin electrons separately. When the SC order is introduced, the ground state is a condensed phase of Cooper pairs each of which is a pair of upspin and down spin electrons. Consequently, the spin Chern number becomes ill-defined for $\Delta_{s} \neq 0$, and the gap closing is not required between the QSH state at $\Delta_{s}=0$ and a trivial state at $\Delta_{s} \neq 0$.

A comment is in order. Here we have assumed the singlet SC order. When we assume the triplet SC order, the energy spectrum is shown to be of the form

$$
E_{\mathrm{BdG}}(k)= \pm \sqrt{\left(\hbar v_{\mathrm{F}}\right)^{2} k^{2}+\left(\sqrt{\lambda_{\mathrm{SO}}^{2}+\Delta_{t}^{2} k^{2}}-|V|\right)^{2}}
$$

The triplet SC parameter $\Delta_{t}$ does not contribute to the gap at $k=0$. The gap closing occurs although this is a topological class change from AII to DIII together with the Bose-Einstein condensation. This is not surprising since the spin-Chern number is well-defined for $\Delta_{t}$ $\neq 0$ because the members of a Cooper pair are either up-spin or down-spin electrons.

Quantum well made of HgTe and CdTe. We present another twodimensional example, which is the Bernevig-Hughes-Zhang (BHZ) model of the QSH system. The BHZ model describes the electronic (a) silicene with

parital SC odering

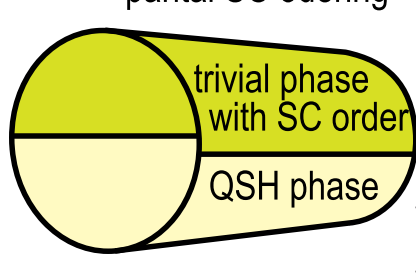

(b) without SC ordering

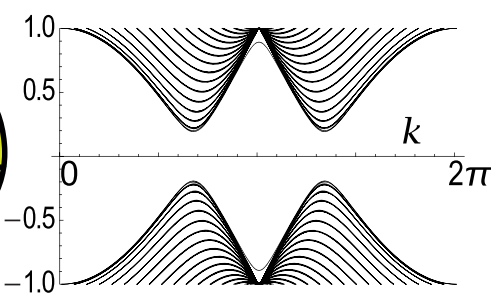

(c) with parital SC odering

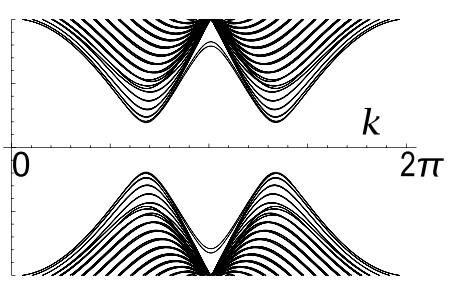

Figure 6 Band structure of silicene in torus geometry. (a) Illustration of silicene in torus geometry with the SC order on one-half of the system. The upper half region is in the trivial phase with the SC order included, while the lower half is in the QSH phase. Band structures of silicene in torus geometry (b) without the SC order and (c) with the the SC order on one-half of the system. The vertical axis is the energy in unit of $t$, while the horizontal axis is the momentum $k$. 
structure of the subband of a quantum well made of $\mathrm{HgTe}$ and $\mathrm{CdTe}^{35}$, which is experimentally verified ${ }^{36}$. A topological phase transition can be induced by changing the thickness of the quantum well.

The Hilbert space is spanned by the four states which are eigenstates of the operator $m_{J},| \pm 1 / 2\rangle$ and $| \pm 3 / 2\rangle$. We present the BHZ Hamiltonian in Supplementary Information. The energy spectrum is determined as

$$
E_{\mathrm{BHZ}}(k)=C-D k^{2} \pm \sqrt{A k^{2}+\left(M-B k^{2}\right)^{2}},
$$

where $A, B, C, D$ are sample-dependent parameters, and $M$ is the Dirac mass. The topological phase transition is known to occur at $M$ $=0$.

To explain it let us slightly simplify the model by choosing $C=0$. Then, the energy spectrum is simplified as

$$
E_{\mathrm{BHZ}}(k)=-D k^{2} \pm \sqrt{A k^{2}+\left(M-B k^{2}\right)^{2}} .
$$

The band gap locates at $k=0$, and is given by $2\left|E_{\mathrm{BHZ}}(0)\right|=2|M|$. We show the band structure of silicene with edges in Figs. 7(c1),(c2),(c3). For $M / B<0$, the system is in the topological phase, as is evidenced by the presence of the gapless edge modes in Fig. 7(c1). As $|M|$ decreases, the gap decreases, and closes at $M=0$ as in Fig. 7 (c2). For $M / B>0$, the gap opens again but no gapless modes appear as in Fig. $7(\mathrm{c} 3)$ : Hence, it is in the trivial phase. Thus, the topological phase transition occurs with gap closing.

We proceed to assume that the singlet Cooper pairs are formed due to the SC proximity effects with the SC gap $\Delta_{s}$. The first pairing is between $|1 / 2\rangle$ and $|-1 / 2\rangle$. The second pairing is between $|3 / 2\rangle$ and $|-3 / 2\rangle$. In general these two gaps are different, but we assume them to be equal for simplicity. The BdG Hamiltonian is derived and given in Supplementary Information. It is diagonalized as

$$
E_{\mathrm{BdG}}(k)= \pm \sqrt{\left(E_{\mathrm{BHZ}}(k)\right)^{2}+\Delta_{s}^{2}},
$$

where each level is two-fold degenerate. The gap does not close when $\Delta_{s} \neq 0$.

We show the topological phase diagram in the $\left(M, \Delta_{s}\right)$ plane, the band gap and the band structure of silicene with edges with straight edge in Fig. 7. Employing the same discussion as in the case of silicene, we find that it is possible to make a topological phase transition without gap closing.

$\mathrm{Cu}$ doped $\mathrm{Bi}_{2} \mathrm{Se}_{3}$. Finally we present a three-dimensional example. $\mathrm{Bi}_{2} \mathrm{Se}_{3}$ is a three-dimensional topological insulator ${ }^{37}$. On the other hand, $\mathrm{Cu}$ doped $\mathrm{Bi}_{2} \mathrm{Se}_{3}{ }^{38-40}$ becomes superconducting at low temperature ${ }^{38-41}$. The topological class change occurs from AII to DIII.

The Hamiltonian is given by ${ }^{42,43}$

$$
H_{\mathrm{BiSe}}=m(k) \tau_{x}+v_{z} k_{z} \tau_{y}+v \tau_{z}\left(k_{x} \sigma_{y}-k_{y} \sigma_{x}\right)-\mu,
$$

with the chemical potential $\mu$, and

$$
m(k)=m_{0}+m_{1} k_{z}^{2}+m_{2}\left(k_{x}^{2}+k_{y}^{2}\right),
$$

where $\sigma_{a}$ and $\tau_{a}$ with $a=x, y, z$ are the Pauli matrices of the spin and the orbital pseudospin, respectively: $\left(v, v, v_{z}\right)$ is the Fermi velocity,
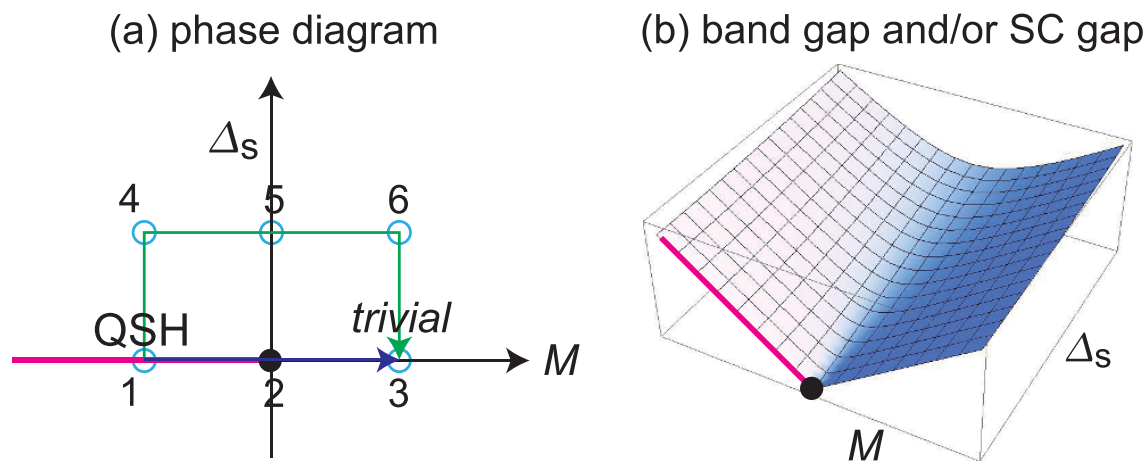

(c) band structure of nanoribbon

(c4)
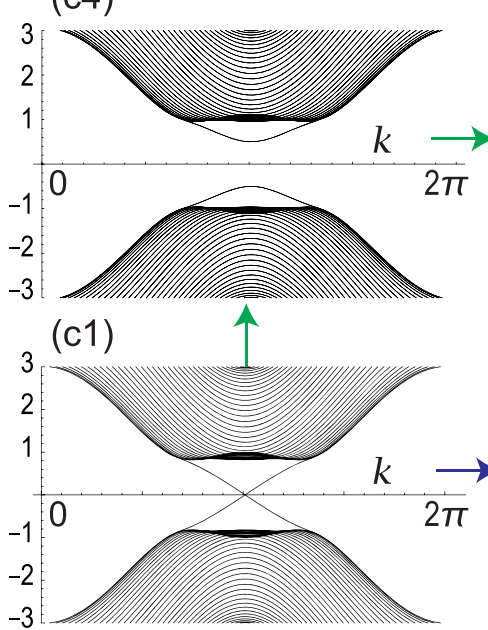

(c5)

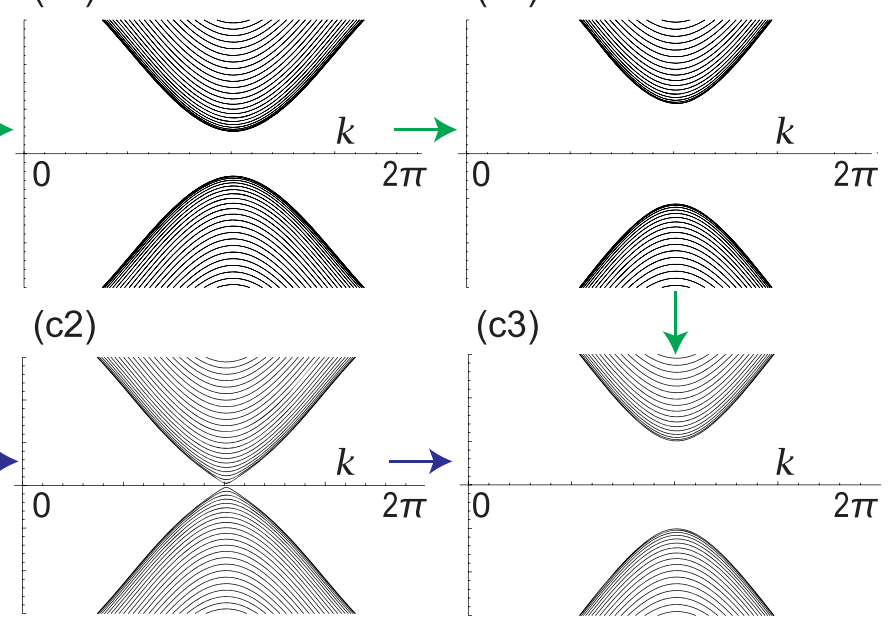

Figure $7 \mid$ Phase diagram and band gap of silicene with Quantum well made of HgTe and CdTe. (a) Topological phase diagram in the $\left(M, \Delta_{s}\right)$ plane. The horizontal axis is the Dirac mass $M$ and the vertical axis is the superconducting gap $\Delta_{s}$. The vertical axis is the energy in unit of $B$. We have set $A=B=1, C$ $=D=0$. See also the caption of Fig. 2 . 
and $m_{i}$ are sample parameters, which satisfy $m_{1} m_{2}>0$. The system is topological when $m_{0} m_{1}<0$ and trivial when $m_{0} m_{1}>0$. The energy spectrum is given by

$$
E_{\mathrm{BiSe}}(k)=-\mu \pm \sqrt{v^{2}\left(k_{x}^{2}+k_{y}^{2}\right)+v_{z}^{2} k_{z}^{2}+m(k)^{2}} .
$$

The band gap locates at the $\Gamma$ point with $k_{x}=k_{y}=k_{z}=0$, where $E_{\text {Bise }}(0)= \pm\left|m_{0}\right|$. The band gap is $2\left|m_{0}\right|$.

We cool the system below the SC transition point. There are four possible superconducting pairing ${ }^{40}$. The simplest one is the intraorbital spin-singlet pairing, which results in trivial superconductor. The other three pairings lead to topological superconductor. We concentrate on the intra-orbital spin-singlet pairing since only this pairing enables the topological class change without gap closing. The BdG Hamiltonian is derived and given in Supplementary Information. It is diagonalized as

$$
E_{\mathrm{BdG}}(k)= \pm \sqrt{\left[E_{\mathrm{BiSe}}(k)\right]^{2}+\Delta_{s}^{2}},
$$

where each level is two-fold degenerate. The gap does not close when $\Delta_{s} \neq 0$. In the same way, the topological class change occurs without gap closing in the three-dimensional space.

Topological superconductor. We have so far studied a model Hamiltonian where the initial and final states are normal state without SC order. Here, we consider a situation where initial and final states are SC states. We consider a model Hamiltonian of twodimensional spin-triplet $p_{x}$-wave superconductor with opposite spin pairing $S_{z}=0$ or spin-singlet $d_{x y}$-wave one. Although the initial Hamiltonian is $4 \times 4$ matrix in the electron-hole and spin spaces, it can be block diagonalized by $2 \times 2$ matrix in the absence of magnetic scattering and spin-orbit coupling. In order to consider the edge state for flat surface parallel to the $y$ direction, we fix momentum $k_{y}$. Then, the original two-dimensional Hamiltonian is reduced to be one-dimensional one. The resulting Hamiltonian can be written as

$$
H=\varepsilon \tau_{z}+\Delta\left(k_{x}\right) \sin k_{x} \tau_{x}
$$

with $\Delta\left(k_{x}\right)=\Delta_{0}$ for $p_{x}$-wave pairing and $\Delta\left(k_{x}\right)=\Delta_{0} \sin k_{y}$ for $d_{x y^{-}}$ wave one, respectively.

The energy spectrum of this Hamiltonian is

$$
E= \pm \sqrt{\varepsilon^{2}+\Delta^{2}\left(k_{x}\right) \sin ^{2} k_{x}}
$$

with $\varepsilon=-t \cos k_{x}-\mu$ and $t>0$ with $\mu=\mu_{0}+t \cos k_{y}$. For $|\mu|<t$, the superconducting state becomes topological with the zero-energy surface Andreev bound state ${ }^{44,45}$. On the other hand, for $|\mu|>t$, it becomes trivial without zero energy ABS. For $|\mu|=t$, the gap closing occurs at $k_{x}=0$ or $k_{x}= \pm \pi$. It has been clarified that the zero energy surface Andreev bound state is protected by the bulk topological number ${ }^{46}$.

Now, let us introduce an additional term $\Delta_{1} \tau_{y}$. Then, the time reversal symmetry is broken and the resulting Hamiltonian becomes

$$
H=\varepsilon \tau_{z}+\Delta\left(k_{x}\right) \sin k_{x} \tau_{x}+\Delta_{1} \tau_{y} .
$$

The energy spectrum is obtained as

$$
E= \pm \sqrt{\varepsilon^{2}+\Delta^{2}\left(k_{x}\right) \sin ^{2} k_{x}+\Delta_{1}^{2}}
$$

There is no gap closing for all $k_{x}$ and $\mu$.

We first start from the topological phase with $\Delta_{1}=0$ with $|\mu|<t$. Next, we switch on $\Delta_{1}$. Then, the system becomes a fully gapped one with time reversal symmetry breaking. We can change $\mu$ from $|\mu|<t$ to $|\mu|>t$. During this change, there is no gap closing. After we switch off $\Delta_{1}$, we reach the topological trivial phase of the original Hamiltonian. One of the example relevant to the intermediate state is $d_{x y}$-wave superconductor with $s$-wave pairing, where the relative phase between these two states is $\pi / 2$. The $\left(d_{x y}+i s\right)$-wave pairing was focused on as the possible surface state of $d_{x y}$-wave pairing in the context of Cuprate ${ }^{47}$. Topological phase transition for fixed momentum has been discussed in superfluid ${ }^{3} \mathrm{He}-\mathrm{B}$ under a magnetic field $h$ parallel to the surface ${ }^{48}$. In this case, helical Andreev bound state remains gapless up to some critical field $h_{c}$ protected by discrete symmetry. There is no bulk gap closing at the transition point $h=h_{c}$.

\section{Discussion}

According to the bulk-edge correspondence, a topological phase transition requires the gap closing and the breakdown of the adiabaticity at the transition point. This is not necessarily the case provided a topological class or symmetry change occurs such that the original set of topological numbers become ill-defined. There exists two possibilities of symmetry change. Both the cases with reduced symmetry and enhanced symmetry have been considered.

When the symmetry is reduced, the target space of the Hamiltonian becomes wider, which enables us to connect two distinct spaces adiabatically. As such an example we have considered a one-dimensional example of polyacetylene by way of the CDW, which induces the class change BDI $\rightarrow$ A. Here, the homotopy class changes from $\pi_{1}\left(S^{1}\right)=\mathbb{Z}$ to $\pi_{1}\left(S^{2}\right)=0$, and the winding number becomes ill-defined. Consequently, we are able to make a topological phase transition (BDI $\rightarrow \mathrm{A} \rightarrow \mathrm{BDI}$ ) to occur without gap closing by switching on and off the CDW. We have also analyzed silicene with the AF order as another example with symmetry reducing. It is interesting that a topological phase transition without gap closing takes place within the same class A. However, there is a symmetry change whether the spin $s_{z}$ is a good quantum number and not. The reduced symmetry case may be regarded as an explicit example of the symmetry protected topological order ${ }^{49-52}$.

When the symmetry is enhanced, the above reasoning no longer follows. As such examples we have considered symmetry increasing transitions (AII $\rightarrow$ DIII) by way of introducing the SC order. Although both the classes AII and DIII are characterized by the $\mathbb{Z}_{2}$ index, their physical meaning is different. The $\mathbb{Z}_{2}$ index is essentially the spin-Chern number in the class AII system, which becomes illdefined as soon as the system moves into the SC phase (DIII) due to the SC order. We have explicitly shown that a topological phase transition (AII $\rightarrow$ DIII $\rightarrow$ AII) may occur without gap closing by switching on and off the SC order.

We also note there is an another mechanism of topological phase transition without gap closing, which is induced by interactions ${ }^{53,54}$. The topological phase transition without gap closing is possible due to the appearnace of zeros in the Green function.

To conclude, we mention the implications of the adiabatic connection between the different sectors of topological number. Up to now only the single-particle Hamiltonian has been considered, but in reality the electron-electron interaction is effective, and the gaps are often induced by the order parameters. Therefore, the character of the quantum critical phenomenon depends strongly on whether the continuous change of the topological number is possible or not. Namely, when we write down the effective action for the quantum phase transition, there are multiple order parameters relevant to the gapless point when the continuous detour is possible to change the topological number. When the multiple topological phase transitions merges at one point, even more interesting quantum critical phenomenon is expected. The global view of the phase diagram taking into account all the possible classes will be an important direction to study the topological quantum transition.

1. Hasan, M. Z. \& Kane, C. Colloquium: Topological insulators. Rev. Mod. Phys. 82, 3045 (2010).

2. Qi, X.-L. \& Zhang, S.-X. Topological insulators and superconductors. Rev. Mod. Phys. 83, 1057 (2011). 
3. Ando, Y. Topological insulator materials. cond-mat/arXiv:1304.5693.

4. Moore, J. E. \& Balents, L. Topological invariants of time-reversal-invariant band structures. Phys. Rev. B 75, 121306(R) (2007).

5. Roy, R. Three dimensional topological invariants for time reversal invariant Hamiltonians and the three dimensional quantum spin Hall effect. Phys. Rev. B 79, 195322 (2009)

6. Tanaka, Y., Sato, M. \& Nagaosa, N. Symmetry and topology in superconductors odd-frequency pairing and edge states. J. Phys. Soc. Jpn. 81, 011013 (2012).

7. Alicea, J. New directions in the pursuit of Majorana fermions in solid state systems. Rep. Prog. Phys. 75, 076501 (2012).

8. Schnyder, A. P., Ryu, S., Furusaki, A. \& Ludwig, A. W. W. Classification of topological insulators and superconductors in three spatial dimensionsn. Phys. Rev. B 78, 195125 (2008).

9. Teo, J. C. Y. \& Kane, C. L. Topological defects and gapless modes in insulators and superconductors. Phys. Rev. B 82, 115120 (2010).

10. Qi, X.-L., Hughes, T. L. \& Zhang, S.-C. Topological field theory of time-reversal invariant insulators. Phys. Rev. B 78, 195424 (2008).

11. Qi, X.-L., Hughes, T. L. \& Zhang, S.-C. Chiral topological superconductor from the quantum Hall state. Phys. Rev. B 82, 184516 (2010).

12. Ezawa, M. Valley-polarized metals and quantum anomalous Hall effect in silicene. Phys. Rev. Lett. 109, 055502 (2012).

13. Ezawa, M. Spin-valleytronics in silicene: quantum-spin-quantum-anomalous Hall insulators and single-valley semimetals. Phys. Rev. B 87, 155415 (2013).

14. Ezawa, M. Photo-induced topological phase transition and single Dirac-cone state in silicene. Phys. Rev. Lett. 110, 026603 (2013).

15. He, J. J. et al. BDI Class Topological superconductors and generating correlated spin currents in auantum anomalous Hall insulators. cond-mat/arXiv:1307.2764.

16. Niemi, A. J. \& Semenoff, G. W. Fermion number fractionizationin quantum field theory. Physics Reports 135, 99 (1986).

17. Su, W. P., Schrieffer, J. R. \& Heeger, A. J. Solitons in polyacetylene. Phys. Rev. Lett. 42, 1698 (1979).

18. Takayama, H. Y., Lin-Liu, Y. R. \& Maki, K. Continuum model for solitons in polyacetylene. Phys. Rev. B 21, 2388 (1980).

19. Monceau, P., Nad, F. Y. \& Brazovskii, S. Ferroelectric Mott-Hubbard phase of organic (TMTTF) $)_{2}$ X conductors. Phys. Rev. Lett. 86, 4080 (2001).

20. Goldstone, J. \& Wilczek, F. Fractional quantum numbers on solitons. Phys. Rev. Lett. 47, 986 (1981).

21. Liu, C.-C., Feng, W. \& Yao, Y. Quantum spin Hall effect in silicene and twodimensional germanium. Phys. Rev. Lett. 107, 076802 (2011).

22. Kane, C. L. \& Mele, E. J. Quantum spin Hall effect in graphene. Phys. Rev. Lett. 95, 226801 (2005)

23. Liu, C.-C., Jiang, H. \& Yao, Y. Low-energy effective Hamiltonian involving spinorbit coupling in silicene and two-dimensional germanium and tin. Phys. Rev. B 84, 195430 (2011).

24. Prodan, E. Robustness of the spin-Chern number. Phys. Rev. B 80, 125327 (2009).

25. Fu, L. \& Kane, C. L. Superconducting proximity effect and Majorana fermions at the surface of a topological insulator. Phys. Rev. Lett. 100, 096407 (2008).

26. Fu, L. \& Kane, C. L. Josephson current and noise at a superconductor/quantumspin-Hall-insulator/superconductor junction. Phys. Rev. B 79, 161408(R) (2009).

27. Alicea, J. Majorana fermions in a tunable semiconductor device. Phys. Rev. B 81, 125318 (2010).

28. Sau, J. D., Lutchyn, R. M., Tewari, S. \& Sarma, S. D. Generic new platform for topological quantum computation using semiconductor heterostructures. Phys. Rev. Lett. 104, 040502 (2010).

29. Lutchyn, R. M., Sau, J. D. \& Sarma, S. D. Majorana fermions and a topological phase transition in semiconductor-superconductor heterostructures. Phys. Rev. Lett. 105, 077001 (2010).

30. Oreg, Y., Refael, G. \& von Oppen, T. Helical liquids and Majorana bound states in quantum wires. Phys. Rev. Lett. 105, 177002 (2010)

31. Meng, L. et al. Buckled silicene formation on $\operatorname{Ir}(111)$. Nano Lett. 13, 685 (2013)

32. Ezawa, M. Topological insulator and helical zero mode in silicene under inhomogeneous electric field. New J. Phys. 14, 033003 (2012).

33. Akhmerov, A. R., Nilsson, J. \& Beenakker, C. W. J. Electrically detected interferometry of Majorana fermions in a topological insulator. Phys. Rev. Lett. 102, 216404 (2009): Linder, J. et al. Unconventional superconductivity on a topological insulator. Phys. Rev. Lett. 104, 067001 (2010).

34. Ezawa, M. Dirac theory and topological phases of silicon nanotube. EPL 98, 67001 (2012)

35. Bernevig, B. A., Hughes, T. L. \& Zhang, S.-C. Quantum spin Hall effect and topological phase transition in HgTe quantum wells. Science 314, 1757 (2006).
36. Koenig, M. et al. Quantum spin Hall insulator state in HgTe quantum wells. Science 318, 766 (2007)

37. Fu, L., Kane, C. L. \& Mele, E. J. Topological insulators in three dimensions. Phys. Rev. Lett. 98, 106803 (2007).

38. Hor, Y. S. et al. Superconductivity in $\mathrm{Cu}_{\mathrm{x}} \mathrm{Bi}_{2} \mathrm{Se}_{3}$ and its implications for pairing in the undoped topological insulator. Phys. Rev. Lett. 104, 057001 (2010).

39. Wray, L. A. et al. Observation of topological order in a superconducting doped topological insulator. Nature Physics 6, 855 (2010)

40. Fu, L. \& Berg, E. Odd-Parity Topological Superconductors: Theory and application to $\mathrm{Cu}_{\mathrm{x}} \mathrm{Bi}_{2} \mathrm{Se}_{3}$. Phys. Rev. Lett. 105, 097001 (2010).

41. Sasaki, S. et al. Topological superconductivity in $\mathrm{Cu}_{x} \mathrm{Bi}_{2} \mathrm{Se}_{3}$. Phys. Rev. Lett. 107, 217001 (2011).

42. Hashimoto, T. et al. Bulk electronic state of superconducting topological insulator. J. Phys. Soc. Jpn. 82, 044704 (2013).

43. Yamakage, A., Yada, K., Sato, M. \& Tanaka, Y. Theory of tunneling conductance and surface-state transition in superconducting topological insulators. Phys. Rev. $B$ 85, 180509(R) (2012).

44. Hu, C. R. Midgap surface states as a novel signature for $\mathrm{d}_{x a}^{2}-\mathrm{x}_{b}^{2}$-wave superconductivity. Phys. Rev. Lett. 72, 1526 (1994).

45. Tanaka, Y. \& Kashiwaya, S. Theory of tunneling spectroscopy of d-wave superconductors. Phys. Rev. Lett. 74, 3451 (1995).

46. Sato, M., Tanaka, Y., Yada, K. \& Yokoyama, T. Topology of Andreev bound states with flat dispersion. Phys. Rev. B 83, 224511 (2011).

47. Matsumoto, M. \& Shiba, H. Coexistence of different symmetry order parameters near a surface in d-wave superconductors I. J. Phys. Soc. Jpn. 64, 3384 (1995).

48. Mizushima, T., Sato, M. \& Machida, K. Symmetry protected topological order and spin susceptibility in superfluid $\mathrm{He}^{3}$-B. Phys. Rev. Lett. 109, 165301 (2012).

49. Gu, Z.-C. \& Wen, X.-G. Tensor-entanglement-filtering renormalization approach and symmetry-protected topological order. Phys. Rev. B 80, 155131 (2009).

50. Turner, A. M., Pollmann, F. \& Berg, E. Topological phases of one-dimensional fermions: An entanglement point of view. Phys. Rev. B 83, 075102 (2011).

51. Chen, X., Liu, Z.-X. \& Wen, X.-G. 2D symmetry protected topological orders and their protected gapless edge excitations. Phys. Rev. B 84, 235141 (2011).

52. Pollmann, F., Berg, E., Turner, A. M. \& Oshikawa, M. Symmetry protection of topological order in one-dimensional quantum spin systems. Phys. Rev. B 85, 075125 (2012).

53. Kitaev, L. \& Fidkowski, A. The effects of interactions on the topological classification of free fermion systems. Phys. Rev. B 81, 134509 (2010).

54. Essin, A. E. \& Gurarie, V. Bulk-boundary correspondence of topological insulators from their respective Green's functions. Phys. Rev. B 84, 125132 (2011).

\section{Acknowledgements}

This work was supported in part by Grants-in-Aid (No. 22740196) for Scientific Research from the Ministry of Education, Science, Sports and Culture of Japan, the Funding Program for World-Leading Innovative RD on Science and Technology (FIRST Program) and by the "Topological Quantum Phenomena" Grant-in Aid (No. 22103005) for Scientific Research on Innovative Areas from the Ministry of Education, Culture, Sports, Science and Technology (MEXT) of Japan.

\section{Author contributions}

M.E. performed the calculations. All authors contributed to the preparation of this manuscript.

\section{Additional information}

Supplementary information accompanies this paper at http://www.nature.com/ scientificreports

Competing financial interests: The authors declare no competing financial interests.

How to cite this article: Ezawa, M., Tanaka, Y. \& Nagaosa, N. Topological Phase Transition without Gap Closing. Sci. Rep. 3, 2790; DOI:10.1038/srep02790 (2013).

cc) (i) $\Theta$ This work is licensed under a Creative Commons Attribution-

BY NC ND NonCommercial-NoDerivs 3.0 Unported license. To view a copy of this license, visit http://creativecommons.org/licenses/by-nc-nd/3.0 


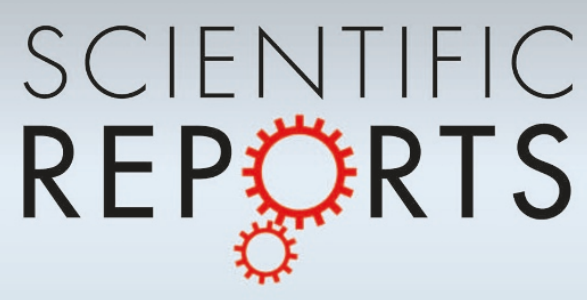

DOI: 10.1038/srep03973

SUBJECT AREAS:

TOPOLOGICAL INSULATORS

PHASE TRANSITIONS AND CRITICAL PHENOMENA

SCIENTIFIC REPORTS:

$3: 2790$

DOI: $10.1038 /$ srep02790

(2013)

Published:

27 September 2013

Updated:

11 February 2014
CORRIGENDUM: Topological Phase Transition without Gap Closing

\section{Motohiko Ezawa, Yukio Tanaka \& Naoto Nagaosa}

This Article contains typographical errors in the Results and Discussion sections, where "A" should read "AI". In the Results section:

"(BDI $\rightarrow \mathrm{A} \rightarrow \mathrm{BDI})$ " should read "(BDI $\rightarrow \mathrm{AI} \rightarrow \mathrm{BDI})$ "

In the Discussion section:

"BDI $\rightarrow$ A" should read "BDI $\rightarrow$ AI"

"(BDI $\rightarrow \mathrm{A} \rightarrow \mathrm{BDI})$ " should read "(BDI $\rightarrow \mathrm{AI} \rightarrow \mathrm{BDI})$ "

In addition, the red line between BDI and A should be between BDI and AI. The correct Figure 1 appears below.

Figure 1

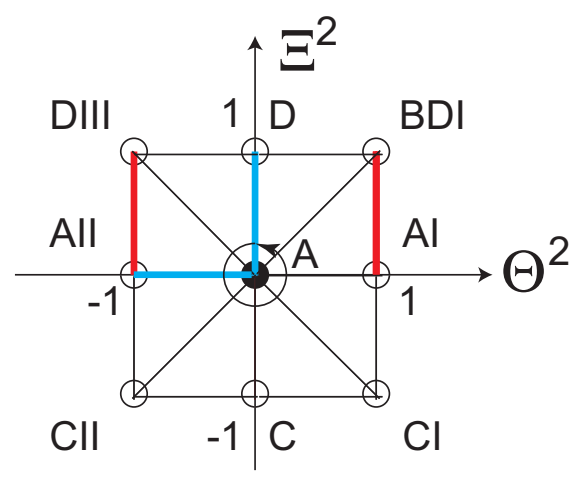

